\title{
Ramos colaterais do arco aórtico e suas principais ramificações em coelho da raça Nova Zelândia (Oryctolagus cuniculus)
}

\author{
Collaterals branches of the aortic arch and its main rami in rabbit (Oryctolagus cuniculus)
}

\author{
Fernanda de Souza $^{\mathrm{I}^{*}}$ Andréia Zechin Bavaresco ${ }^{\mathrm{I}}$ Rui Campos $^{\mathrm{II}}$
}

RESUMO

Neste trabalho, sistematizaram-se os ramos colaterais do arco aórtico e suas principais ramificações, utilizando 30 coelhos da raça Nova Zelândia. O sistema arterial foi preenchido com látex corado em vermelho através da aorta torácica no sentido contrário ao fluxo sanguíneo e fixado em uma solução aquosa de formaldeído a $20 \%$. Os ramos colaterais do arco aórtico foram o tronco braquiocefálico e a artéria subclávia esquerda. O tronco braquiocefálico emitiu as artérias carótidas comuns esquerda e direita, continuando-se como artéria subclávia direita. Esta emitiu, de medial para lateral, as artérias: vertebral, tronco comum cervical superficial-profunda e tronco costocervical-torácica interna. Já a artéria subclávia esquerda emitiu as artérias: vertebral, intercostal suprema, torácica interna, escapular dorsal e tronco comum cervical superficial-profunda.

Palavras-chave: arco aórtico, vascularização, coelhos.

\section{ABSTRACT}

In this study, the collaterals branches of the aortic arch and its main rami were systematized in 30 New Zealand rabbits (Oryctolagus cuniculus). The arterial system was filled with red latex in the opposite direction to blood flow, and fixed in $20 \%$ formaldehyde solution. The collaterals branches of the aortic arch were the brachiocephalic trunk and the left subclavian artery. The brachiocephalic truk emitted the left and the right common carotid arteries, continuing as the right subclavian artery. This emitted, from medial to lateral, the arteries: vertebral, superficialdeep cervical common trunk and costocervical-internal thoracic trunk. Already the left subclavian artery emitted the arteries: vertebral, supreme intercostal, internal thoracic, dorsal scapular and superficial-deep cervical common trunk.

Key words: aortic arch, vascularization, rabbits.

\section{INTRODUÇÃO}

O coelho doméstico Oryctolagus cuniculus é um lagomorfo da família Leporidea, que pertenceu por muito tempo à ordem dos roedores (ANDRADE, 2002). É um animal que se destaca pela intensidade do seu uso como animal de experimentação, o qual, ao longo de toda a história da medicina experimental, seja no âmbito da cirurgia, da farmacologia ou da imunologia, vem desempenhando papel de indiscutível relevância (HARKNESS \& WAGNER, 1993). Informações morfológicas sobre o coelho são raras, sendo encontrados dois relatos sobre as ramificações do arco aórtico nessa espécie. Para confronto de resultados, utilizaram-se referências de alguns roedores na discussão, como: chinchila, nutria, porco-espinho, paca, cobaia, capivara e mocó.

O presente trabalho tem como objetivo sistematizar e descrever os ramos colaterais do arco aórtico e suas principais ramificações em coelho da raça Nova Zelândia, estabelecendo um modelo padrão e as principais variações nessa espécie.

\section{MATERIAL E MÉTODOS}

Foram utilizados trinta coelhos da raça Nova Zelândia (Oryctolagus cuniculus), machos e fêmeas, adultos, provenientes de criatórios da Região Metropolitana de Porto Alegre, RS.

\footnotetext{
IPrograma de Pós-graduação em Ciências Veterinárias (PPGCV), Faculdade de Veterinária, Universidade Federal do Rio Grande do Sul (UFRGS), 91540-000, Porto Alegre, RS, Brasil. E-mail: fesouzavet@yahoo.com.br. *Autor para correspondência.

ILLaboratório de Anatomia Animal, Departamento de Ciências Morfológicas, Instituto de Ciências Básicas e da Saúde (ICBS), UFRGS, Porto Alegre, RS, Brasil. 
Os animais foram contidos e eutanasiados com injeção de T 61 (3ml animal-1) pela via intrapulmonar. A pele foi rebatida, a cavidade torácica aberta em plastrão, o saco pericárdio aberto, a aorta torácica canulada em contrafluxo e as duas veias cavas craniais seccionadas próximas ao coração.

O sistema foi lavado com solução salina aquosa a 0,9\% e heparina (5000UI animal-1) na quantidade de $120 \mathrm{ml}$ animal $^{-1}$ e preenchido com látex 603 corado em vermelho. Os animais permaneceram imersos em água corrente para a polimerização do látex por uma hora e meia, sendo em seguida, seccionada a coluna vertebral na altura das últimas vértebras torácicas. As peças foram fixadas em formaldeído a $20 \%$ por sete dias e, transcorrido esse período, foram dissecadas as artérias do espaço mediastínico cranial e pescoço. Desenhos esquematizados de todas as peças foram realizados com auxílio de lupa para a confecção dos resultados. Alguns exemplares foram fotografados para documentação e os vasos nomeados conforme a NOMINA ANATOMICA VETERINARIA (2005). A análise estatística dos resultados constou da aplicação do cálculo de percentagem.

\section{RESULTADOS}

A aorta do coelho nasceu do ventrículo esquerdo e projetou-se craniodorsolateralmente à esquerda, formando o arco aórtico em todas as preparações. O arco aórtico emitiu como ramos colaterais em sequência o tronco braquiocefálico e a artéria subclávia esquerda em $80 \%$ das amostras
(Figuras 1 A e 2 A). Em 13,4\% das peças, a sequência dos ramos foi o tronco braquiocefálico, a artéria carótida comum esquerda e a artéria subclávia esquerda (Figuras 1 B e 2 B). Já em 3,3\% dos achados, a sequência dos ramos colaterais do arco aórtico foi o tronco braquiocefálico, tronco comum cervical superficial-profunda e artéria subclávia esquerda. Em 3,3\% dos casos, a sequência dos ramos colaterais do arco aórtico foi o tronco braquiocefálico, a artéria carótida comum esquerda, o tronco comum cervical superficial-profunda e a artéria subclávia esquerda.

O tronco braquiocefálico emitiu em 83,3\% dos casos uma artéria carótida comum esquerda, e milímetros depois uma artéria carótida comum direita, continuando-se para a direita como artéria subclávia direita (Figuras 1 A e 2 A). Já em 16,7\% das preparações, o tronco braquiocefálico lançou, alguns milímetros depois de sua origem, uma artéria carótida comum direita e continuou-se como artéria subclávia direita (Figuras 1 B e 2 B).

As artérias carótidas comuns direita e esquerda ascenderam o pescoço, acompanhando a traqueia, lateralmente, alcançando a base do crânio dividindo-se, na altura da alça do nervo hipoglosso, em artéria carótida interna, que participava da irrigação do encéfalo do coelho, continuando naturalmente como artéria carótida externa, a qual se distribuiu com seus ramos na face. A artéria carótida interna direita esteve presente como ramo da artéria carótida comum direita em 96,7\% dos achados. Em 3,3\% das peças, a artéria carótida interna direita esteve ausente, porém, a artéria do antímero esquerdo originou, na base da hipófise, um ramo que a substituiu. Em todas

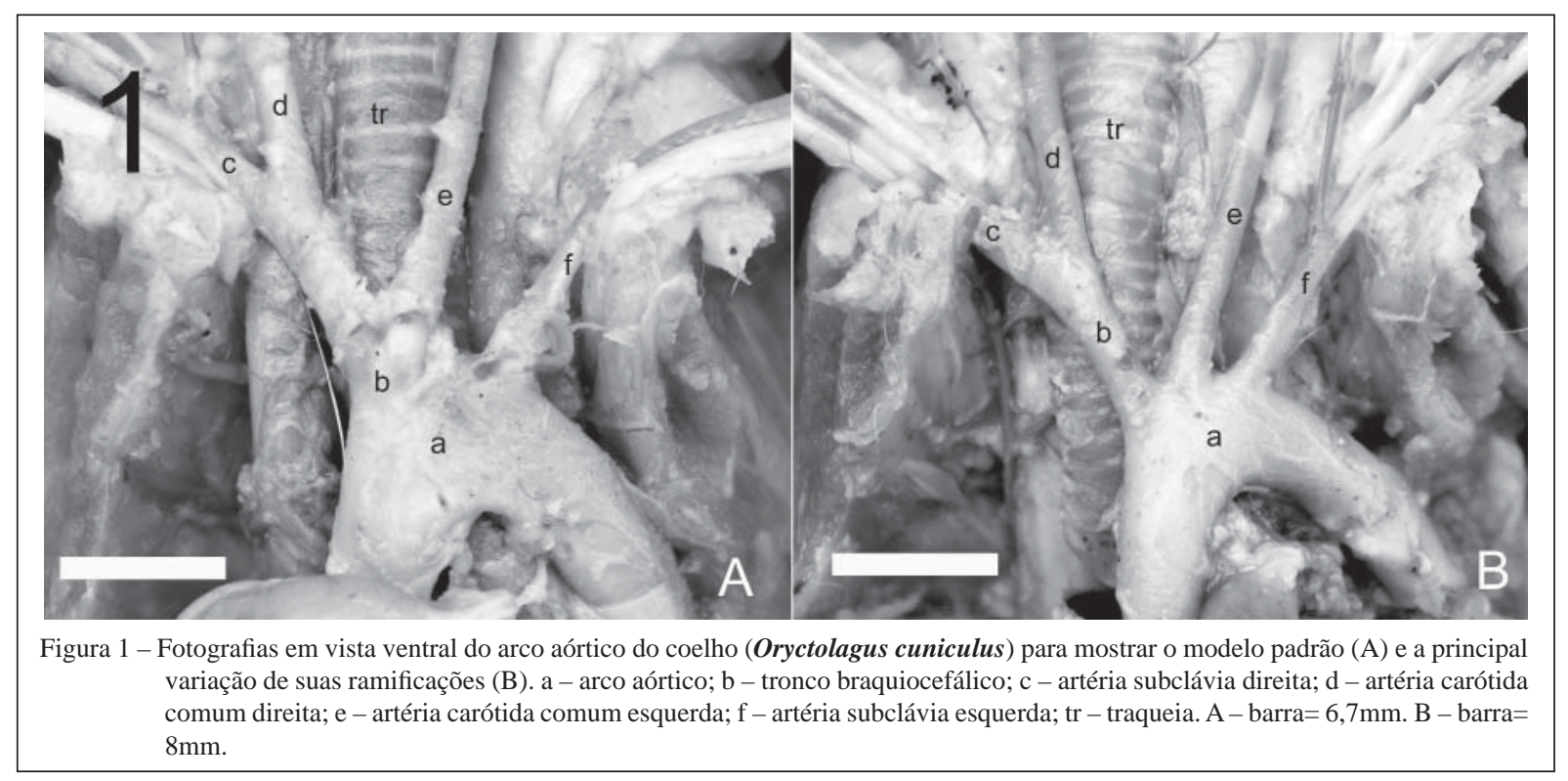

Ciência Rural, v.43, n.12, dez, 2013. 


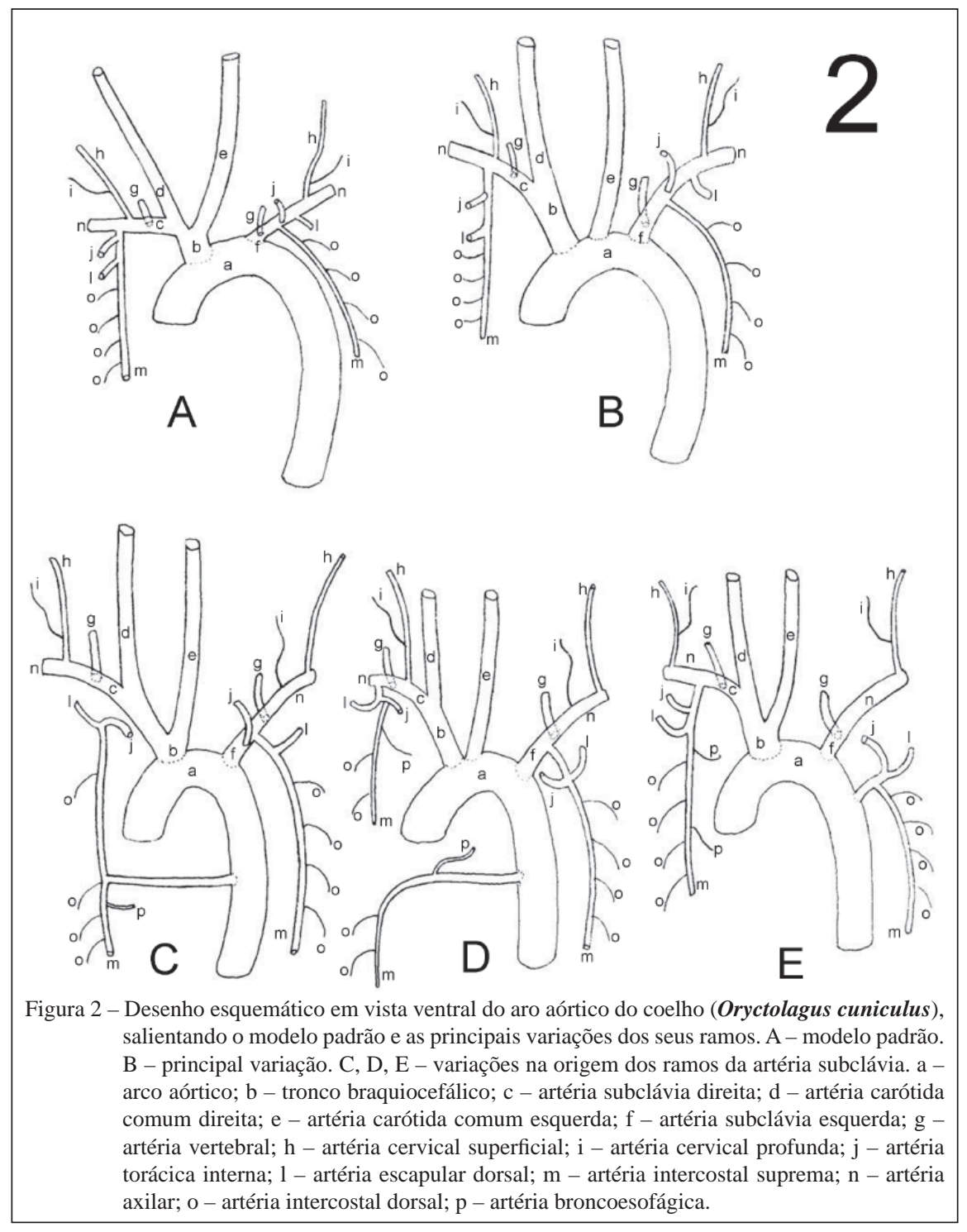

as preparações, a artéria carótida interna esquerda esteve presente como ramo da artéria carótida comum esquerda, indo cooperar com a vascularização do encéfalo.

A artéria subclávia direita emitiu, de medial para lateral, como ramos colaterais principais, as artérias: vertebral como primeiro ramo em 53,3\%; tronco comum cervical superficial-profunda como segundo em 33,3\%; e tronco costocervical-torácica interna como terceiro em 50\% dos achados (Figura $2 \mathrm{~A}$ ). Já a artéria subclávia esquerda emitiu, também de medial para lateral, como ramos colaterais principais, as artérias: vertebral como primeiro ramo em 43,3\%; intercostal suprema como segundo em 23,3\%; torácica interna como terceiro em $26,6 \%$; escapular dorsal como quarto em $20 \%$; e tronco comum cervical superficial-profunda como quinto ramo em 36,6\% das preparações (Figura 2 B).

Os vasos em tronco ou isoladamente apresentaram um território constante de vascularização arterial, ou seja: a artéria vertebral projetou-se craniodorsalmente, adentrando ao canal transversal no forame transverso da sexta vértebra cervical. Percorreu o canal transversal até atingir o atlas, onde contornou a incisura alar adentrando ao canal vertebral pelo forame vertebral lateral. Anastomosou-se com sua homóloga contralateral na base da entrada do forame magno, formando a artéria basilar que emitiu ramos colaterais responsáveis pela vascularização do rombencéfalo. O tronco cervical superficial-profunda emitiu como ramo mais calibroso a artéria cervical superficial que percorreu a face lateral superficial do 
pescoço, irrigando a musculatura ventral deste e a musculatura extrínseca do membro torácico na parte cervical. Seu ramo de menor calibre, a artéria cervical profunda, ramificou-se um pouco mais profundamente no pescoço na musculatura extrínseca do membro torácico, mais caudal. O tronco costocervical foi um vaso volumoso, cujos ramos irrigaram a maior parte da região cervical dorsal e as partes dorsocraniais da parede torácica. Ele originou as artérias escapular dorsal e intercostal suprema. A artéria escapular dorsal irrigou a face profunda da musculatura extrínseca do membro torácico. A artéria intercostal suprema acompanhou o colo das quatro primeiras costelas, emitindo as quatro primeiras artérias intercostais dorsais e a artéria broncoesofágica. Já a artéria torácica interna percorreu caudalmente entre as cartilagens costais e o músculo transverso torácico emitindo todos os ramos intercostais ventrais, terminandose no processo xifóide nas artérias musculofrênica e epigástrica cranial.

As ocorrências dos ramos colaterais da artéria subclávia, à direita, foram: a artéria vertebral, sendo emitida individualmente como primeiro ramo em 53,3\% (Figuras 2 A, 2 B, 2 C e 2 E), como segundo em 43,3\% (Figura 2 D) e como terceiro ramo em $3,3 \%$ dos casos. O tronco cervical superficialprofunda, emitido como primeiro ramo da artéria subclávia direita em 36,7\% (Figuras 2 C e 2 D), como segundo em 33,3\% e como terceiro ramo em $26,7 \%$ das peças. Nos restantes $3,3 \%$ dos casos não houve a formação de tronco, sendo a artéria cervical profunda emitida isolada como segundo ramo da artéria subclávia direita e a artéria cervical superficial foi originada da artéria axilar (Figura 2 E). O tronco costocervical, formado pelas artérias escapular dorsal e intercostal suprema, mais a artéria torácica interna foram emitidos da artéria subclávia em tronco comum, à direita, em $80 \%$ dos casos; sendo terceiro ramo em 50\% (Figuras 2 A e 2 B), segundo em 20\% (Figura 2 E) e primeiro ramo em $10 \%$ dos casos. Nos $20 \%$ restantes, esses ramos colaterais foram emitidos em uma sequência de vasos isolados ou formando troncos compostos de formas variadas. Em uma peça, houve a formação do tronco costocervical, sendo a artéria torácica interna emitida isolada como quarto ramo da artéria subclávia direita. Já em outra amostra, dentro dos $20 \%$, as três artérias foram emitidas de forma isolada, sendo a artéria intercostal suprema o terceiro ramo, a artéria torácica interna o quarto e a artéria escapular dorsal o quinto ramo. Ainda em quatro peças dentro dos $20 \%$, do final do arco aórtico originou-se um tronco que se projetou para o antímero direito emitindo as artérias intercostal suprema, escapular dorsal e torácica interna (Figuras $2 \mathrm{C}$ e 2 D). Em uma destas quatro peças, houve a formação de um tronco comum torácica internaescapular dorsal como terceiro ramo, sendo a artéria intercostal suprema emitida da artéria torácica interna como se fosse uma artéria vertebral torácica (Figura 2 D). Também ocorreu a formação de outro tronco isolado originado da aorta torácica que atravessou a linha mediana para o antímero direito, lançando nesse percurso uma artéria broncoesofágica direita, seguindo caudalmente como artéria intercostal suprema (Figura 2 D). Nos outros três casos, o tronco isolado originado da aorta no fim do arco aórtico emitiu, no antímero direito, caudalmente a artéria intercostal suprema, a qual lançou uma artéria broncoesofágica direita, e cranialmente um segundo tronco que originou a artéria escapular dorsal e a artéria torácica interna direitas (Figura 2 C).

Já à esquerda, as ocorrências dos ramos colaterais da artéria subclávia foram: a artéria vertebral, sendo primeiro ramo em 43,3 \% das peças (Figuras $2 \mathrm{~A}$, 2 B e 2 E), segundo em 23,3\% (Figura 2 D), quarto em $20 \%$ e terceiro ramo em $13,3 \%$ das amostras (Figura $2 \mathrm{C}$ ). O tronco cervical superficial-profunda, que foi quinto ramo em 36,7\% dos casos (Figuras 2 A e 2 B), terceiro em 16,7\%, quarto em 13,3\% e segundo ramo em 3,3\% das amostras. Em 6,7\%, o tronco cervical superficial-profunda originou-se do arco aórtico entre o tronco braquiocefálico e artéria subclávia esquerda. Em 3,3\% das peças, o tronco cervical superficial-profunda foi emitido da artéria escapular dorsal e em outros 3,3\% era ramo da artéria torácica interna. Nos restantes $16,7 \%$ das peças onde não houve a formação de tronco: em duas dessas amostras a artéria cervical profunda apresentouse como terceiro ramo; em outras duas peças como quarto ramo; e em um dos casos como quinto ramo da artéria subclávia esquerda. Em todos esses casos em que a artéria cervical profunda esteve isolada, à esquerda, a artéria cervical superficial foi emitida da artéria axilar (Figuras 2 C, 2 D e 2 E). A artéria torácica interna esquerda foi lançada de forma isolada da artéria subclávia esquerda em 66,7\% dos casos, sendo terceiro ramo em 26,7\% (Figuras 2 A e 2 B), segundo em 16,7\%, primeiro em $13,3 \%$ (Figura 2 C) e quarto ramo em $10 \%$ das amostras. Em 30\% dos casos, a artéria torácica interna esquerda originou-se da artéria subclávia esquerda em tronco comum com as artérias do tronco costocervical de forma variada, conforme a tabela 1 . A artéria intercostal suprema esquerda foi emitida da artéria subclávia esquerda de forma isolada em 53,3\% das amostras, sendo o primeiro ramo em 23,3\%, o segundo em 23,3\% (Figuras 2 A e 2 B) e o terceiro 
Tabela 1 - Frequência de variação na formação de troncos comuns entre as artérias torácica interna, intercostal suprema e escapular dorsal como ramo colateral da artéria subclávia esquerda.

\begin{tabular}{lcc}
\hline Tronco comum & Como $1^{\circ}$ ramo & Como $2^{\circ}$ ramo \\
\hline Aa. I.S. \& T.I. & $3,3 \%$ & - \\
Aa. I.S., T.I. \& E.D. & $13,3 \%$ & $10 \%$ \\
Aa. E.D. \& T.I. & - & $3,3 \%$ \\
\hline
\end{tabular}

Aa. I.S. \& T.I.= artérias intercostal suprema e torácica interna; Aa. I.S., T.I. \& E.D.= artérias intercostal suprema, torácica interna e escapular dorsal; Aa. E.D. \& T.I.= artérias escapular dorsal e torácica interna.

em 6,7\% das peças. Em 43,3\% dos casos, a artéria intercostal suprema originou-se da artéria subclávia esquerda em tronco comum com as artérias torácica interna e escapular dorsal esquerdas de forma variada, conforme a tabela 1. A artéria escapular dorsal foi emitida da artéria subclávia esquerda de forma isolada em 53,3\% das amostras, sendo terceiro ramo em 26,7\%, quarto em 20\% (Figuras 2 A e 2 B) e segundo ramo em $6,7 \%$ das preparações. Em 43,3\% dos casos, a artéria escapular dorsal originou-se da artéria subclávia esquerda em tronco comum com as artérias torácica interna e intercostal suprema esquerdas de forma variada, conforme a tabela 1 .

Em 3,3\% das peças, a aorta, no final de seu arco, emitiu um tronco curto que lançou caudalmente uma artéria intercostal suprema esquerda e lateralmente outro tronco que originou as artérias torácica interna e escapular dorsal esquerdas (Figura $2 \mathrm{E}$ ).

\section{DISCUSSÃO}

O arco aórtico em chinchila (ARAÚJO et al., 2004), nutria (CAMPOS et al., 2010), paca (OLIVEIRA et al., 2001), mocó (MAGALHÃES et al., 2007) e porquinho-da-índia (KABAK \& HAZIROGLU, 2003) projetou-se para a esquerda e emitiu como ramos colaterais, em sequência, o tronco braquiocefálico e a artéria subclávia esquerda na maioria dos casos, coincidindo com o encontrado em coelho. Também em chinchila (ARAÚJO et al., 2004) e nutria (CAMPOS et al., 2010), observou-se a emissão do tronco braquiocefálico, seguido da artéria carótida comum esquerda e da artéria subclávia esquerda em algumas peças, sendo isso semelhante com o achado em coelho. Ainda, foi encontrada neste estudo, a emissão do tronco comum cervical superficial-profunda do arco aórtico do coelho na minoria dos casos, o que não foi observado em outras espécies estudadas. Em capivara, do arco aórtico foi lançado apenas o tronco braquiocefálico (CULAU et al., 2007), não sendo isso constatado em coelho.

Do tronco braquiocefálico foram emitidas, em sequência, as artérias carótida comum esquerda, carótida comum direita e subclávia direita na maioria dos achados em chinchila (ARAÚJO et al., 2004), nutria (CAMPOS et al., 2010), mocó (MAGALHÃES et al., 2007), porquinho-da-índia (KABAK \& HAZIROGLU, 2003) e paca (OLIVEIRA et al., 2001), como também em coelho. Já em poucas peças em chinchila (ARAÚJO et al., 2004) e em quase metade das amostras em nutria (CAMPOS et al., 2010), do tronco braquiocefálico foram lançadas apenas as artérias carótida comum direita e subclávia direita, o que também foi visto em coelho em algumas amostras. ALBUQUERQUE et al. (1987), evidenciaram que em coelho, o tronco braquiocefálico lançou a artéria carótida comum esquerda, seguida de ramo de origem das artérias carótida comum direita e subclávia direita, sendo a artéria subclávia esquerda lançada do arco aórtico, em quase todas as amostras examinadas, concordando com o presente estudo também em coelho. Porém, em alguns casos, esses autores encontraram o tronco braquiocefálico emitindo a artéria subclávia direita seguida de um tronco bicarotídeo, fato não observado nos coelhos analisados. Em mocó, na maioria dos achados houve a formação do tronco bicarotídeo depois da origem da artéria subclávia direita (MAGALHÃES et al., 2007). CULAU et al. em (2007), encontraram na maioria das capivaras estudadas, um tronco braquiocarotídeo sendo lançado do tronco braquiocefálico após a emissão das artérias subclávia esquerda e carótida comum esquerda. Esse tronco, que deu origem às artérias carótida comum direita e subclávia direita, não foi identificado em coelho.

Para BARONE (1996), as artérias carótidas comuns em coelho, na altura da alça do nervo hipoglosso, bifurcaram-se nas artérias carótidas externa e interna, sendo que a primeira foi em direção à face, e a segunda cooperou com a irrigação do encéfalo; o que coincide com os resultados deste trabalho, exceto em um caso, em que a artéria carótida interna direita esteve ausente, porém, a artéria carótida interna contralateral originou na base da hipófise um ramo que a substituiu. Já em nutria (CAMPOS et al., 2010), as artérias carótidas comuns dividiram-se nas artérias carótida externa e occipital, sendo que esta última emitiu uma fina artéria carótida interna que não participou da irrigação encefálica. Em chinchila (ARAÚJO et al., 2004), em apenas dois casos, à esquerda, a artéria carótida interna chegou a participar da irrigação encefálica.

Ciência Rural, v.43, n.12, dez, 2013. 
Com relação à ramificação da artéria subclávia, em paca (OLIVEIRA et al., 2001) e em porquinho-da- índia (KABAK \& HAZIROGLU, 2003) essa artéria ramificou-se nas artérias vertebral, tronco costocervical, cervical superficial, torácica interna e axilar. Já em chinchila (ARAÚJO et al., 2004) e nutria (CAMPOS et al., 2010), como também neste estudo em coelho, houve uma variação significativa quanto à sequência de emissão desses vasos nos dois antímeros. Em chinchila (ARAÚJO et al., 2004), a maior prevalência de emissão da artéria subclávia direita, de medial para lateral, foi: as artérias vertebral, escapular dorsal, tronco comum torácica interna-vertebral torácica e tronco comum cervical superficial-profunda. Em nutria (CAMPOS et al., 2010), não houve a formação do tronco comum entre as artérias torácica interna e vertebral torácica, sendo emitidos cinco vasos da artéria subclávia direita. Já em coelho, a sequência de ramos encontrada foi: as artérias vertebral, tronco comum cervical superficialprofunda e tronco costocervical-torácica interna.

A artéria subclávia esquerda em chinchila (ARAÚJO et al., 2004) lançou os mesmos quatro vasos do antímero direito, havendo apenas alteração na sequência de emissão: tronco comum torácica internavertebral torácica, vertebral, escapular dorsal e tronco comum cervical superficial-profunda. Em nutria (CAMPOS et al., 2010), foram emitidas as artérias, de medial para lateral: vertebral torácica, torácica interna, vertebral, tronco escapular dorsal com tronco comum cervical superficial-profunda. Já em coelho, foi observada a seguinte sequência de vasos: vertebral, intercostal suprema, torácica interna, escapular dorsal e tronco comum cervical superficial-profunda.

Diferentemente do encontrado em nutria (CAMPOS et al., 2010), em que na minoria dos casos houve formação de um tronco comum entre as artérias cervical superficial e profunda, tanto à direita quanto à esquerda; em coelho foi encontrado este tronco comum em quase todos os casos, assim como em chinchila (ARAÚJO et al., 2004).

Segundo BARONE (1996), a artéria intercostal suprema teve essa denominação em coelho, devido ao fato desta originar as quatro primeiras artérias intercostais dorsais, diferente do que ocorre em carnívoros, chinchila (ARAÚJO et al., 2004), nutria (CAMPOS et al., 2010), paca (OLIVEIRA et al., 2001) e porquinho-da-índia (KABAK \& HAZIROGLU, 2003), nos quais as três primeiras artérias intercostais dorsais foram emitidas diretamente do tronco costocervical. Por isso, não foi considerada uma verdadeira intercostal suprema, recebendo o nome de artéria vertebral torácica. Porém, em um caso à direita em coelho, houve a formação de um tronco comum torácica interna-escapular dorsal, sendo a artéria intercostal suprema emitida da artéria torácica interna com o comportamento de uma artéria vertebral torácica. Nesse mesmo caso, também se originou da aorta torácica outro tronco isolado, que atravessou a linha mediana e seguiu como uma artéria intercostal suprema caudalmente.

Não foi encontrada na literatura pesquisada nenhuma citação de casos de formação de tronco originado no final do arco aórtico, o qual era direcionado para o antímero direito ou esquerdo e emitia as artérias intercostal suprema, escapular dorsal e torácica interna, como achado em alguns dos coelhos analisados.

\section{CONCLUSÃO}

Com base nos resultados obtidos podese concluir que o padrão dos ramos colaterais do arco aórtico em coelho foi, em sequência, o tronco braquiocefálico e a artéria subclávia esquerda, enquanto que as variações encontradas foram tronco braquiocefálico, artéria carótida comum esquerda e artéria subclávia esquerda; e dois casos em que o tronco cervical-superficial-profunda também era ramo colateral do arco. Os ramos colaterais da artéria subclávia direita foram mais constantes do que os ramos da artéria subclávia esquerda, tendo-se encontrado mais variações no antímero esquerdo.

\section{AGRADECIMENTO}

À Coordenação de Aperfeiçoamento de Pessoal de Nível Superior (CAPES), pela bolsa de estudo.

\section{COMITÊ DE ÉTICA E BIOSSEGURANÇA}

O experimento foi aprovado pela Comissão de Ética na Utilização de Animais (CEUA) da Universidade Federal do Rio Grande do Sul (UFRGS), Brasil, sob número 19383, em 30 de setembro de 2010 .

\section{REFERÊNCIAS}

ALBUQUERQUE, J.F.G. et al. Contribuição ao estudo dos Colaterais Calibrosos do Arco Aórtico no Coelho (Oryctolagus cuniculus, LINNAEUS 1758) da Raça Nova Zelândia. Ars Veterinária UNESP, v.3, p.1-4, 1987.

ANDRADE, A. et al. (Org.) Animais de laboratório - criação e experimentação. Rio de Janeiro: Fiocruz, 2002. 388p.

ARAÚJO, A.C.P. et al. Ramos colaterais do arco aórtico e suas principais ramificações em chinchila (Chinchilla lanigera). Revista Portuguesa de Ciências Veterinárias, v.99, p.53- 
58, 2004. Disponível em: <http://www.fmv.utl.pt/spcv/PDF/ pdf3_2004/549_53_58.pdf>.Acesso em: 20 jul. 2012.

BARONE, R. Anatomie comparée des mammifères domestiques: Angiologie. Paris: Vigot, 1996. 5v.

CAMPOS, R. et al. Ramos colaterais do arco aórtico e suas principais ramificações em nutria (Myocastor coypus). Acta Scientiae Veterinariae, v.38, p.139-146, 2010. Disponível em: <http://hdl.handle.net/10183/29229>. Acesso em: 23 jul. 2012.

CULAU, P.O.V. et al. Colaterais do arco aórtico da capivara (Hydrochoerus hydrochaeris). Acta Scientiae Veterinariae, v.35, p.89-92, 2007. Disponível em: <http://hdl.handle. net/10183/20565>. Acesso em: 23 jul. 2012.

HARKNESS, J.E.; WAGNER, J.E. Biologia e clínica de coelhos e roedores. São Paulo: Roca, 1993. 238p. International Committee On Veterinary Gross Anatomical Nomenclature. Nomina anatomica veterinaria. New York: Nomina anatomica veterinaria, 2005. 190p.
KABAK, M.; HAZIROGLU, R.M. Subgross investigation of vessels originating from arcus aortae in guinea-pig (Cavia porcellus). Anatomia, Histologia, Embryologia, v.32, p.362-366, 2003. Disponível em: <http://onlinelibrary.wiley.com/doi/10.1111/ j.1439-0264.2003.00497.x/abstract;jsessionid=8993E630F3CCD E0CDBA50EDBD5D2821A.d01t02?deniedAccessCustomisedM essage $=$ \&userIsAuthenticated=false $>$. Acesso em: 24 jul. 2012. doi: 10.1111/j.1439-0264.2003.00497.x.

MAGALHÃES, M. et al. Ramos do arco aórtico no mocó (Kerodon rupestris). Revista Portuguesa de Ciências Veterinárias, v.102, p.49-52, 2007. Disponível em: <http://www.fmv.utl.pt/spcv/PDF/ pdf6_2007/49-52.pdf>. Acesso em 24 jul. 2012.

OLIVEIRA, F.S. et al. Gross anatomical study of the aortic ARC branches of the paca (Agouti paca, Linnaeus, 1766). Brazilian Journal of Veterinary Research and Animal Science, v.38, p.103-105, 2001. Disponível em: <http://revistas.usp.br/bjvras/ article/view/5891>. Acesso em: 24 jul. 2012. doi: 10.1590/S141395962001000300001 